\title{
Long-term follow-up for lumbar intrathecal baclofen catheters placed using the paraspinal subfascial technique
}

\author{
Saumitra K. Thakur, BA, Benjamin A. Rubin, MD, and David H. Harter, MD \\ Department of Neurosurgery, New York University Langone Medical Center, New York, New York
}

OBJECTIVE Intrathecal baclofen (ITB) is a valuable therapeutic option for patients with spasticity and dystonia. The techniques that place an ITB pump catheter into the subcutaneous fat of a lumbar incision are well described. Because patients who require ITB often have low body fat content, they may be predisposed to catheter-related complications. The senior author used a novel technique to place the catheter in a paraspinal subfascial fashion, and the short-term results were previously published. That study demonstrated no development of hardware erosions, catheter migrations, or CSF leaks within an average follow-up of 5 months. This study followed up on those initial findings by looking at the long-term outcomes since this technique was introduced.

METHODS Using the institutional review board-approved protocol, the electronic medical records were reviewed retrospectively for all patients who underwent paraspinal subfascial catheter placement by the senior author. Patients received follow-up with the surgeon at 2 weeks postoperatively and were followed routinely by their physiatrist thereafter.

RESULTS Of the 43 patients identified as having undergone surgery by the senior author using the paraspinal subfascial technique between July 2010 and February 2014, 12 patients (27.9\%) required reoperation. There were 5 patients $(11.6 \%)$ who had complications related to the catheter or lumbar incision. No hardware erosions or CSF leaks were identified. These patients received a median follow-up of 3.0 years, with 30 of 43 patients receiving follow-up over 2.0 years.

CONCLUSIONS This follow-up study suggests that the technique of paraspinal subfascial catheter placement translates to long-term decreases in CSF leakage and complications from erosion, infection, and also catheter malfunctions. It does not seem to affect the overall rate of complications.

http://thejns.org/doi/abs/10.3171/2015.7.PEDS15137

KEY WORDS paraspinal; subfascial; ITB; catheter placement; technique; intrathecal baclofen

I NTRATHECAL baclofen (ITB) therapy has become a valuable therapy for medically refractory spasticity and dystonia in both adults and children. The most common diagnoses that lead to spasticity and may be indications for ITB therapy include cerebral palsy $(\mathrm{CP})$, traumatic brain injury, spinal cord injury, diffuse anoxic brain injury, multiple sclerosis, and various hereditary conditions.5 Additionally, ITB is very effective as a palliative treatment for improving patient posture and easing general caregiving. ${ }^{15}$ In a review article by the American Academy for Cerebral Palsy and Developmental Medicine, all 17 cited studies on ITB use in patients with spastic cerebral palsy demonstrated a significant reduction in muscle tone, especially in the lower extremities. ${ }^{7}$ Furthermore, the efficacy of ITB for treating spasticity subsequently reduces the need for orthopedic surgery in patients with spasticity. ${ }^{1,4,9,11}$
Because ITB therapy has been used so successfully, ITB pump implantation has become a common procedure to perform. As with any implantable device, there can be complications. The common surgical complications of ITB pump placement include device malfunctions, acute pump failures, catheter breaks and fractures, disconnections, and catheter kinking. Additional complications include pump hypermobility, skin erosion, persistent CSF leakage, and infection. ${ }^{1,4,6,10-12}$ Patients who require ITB therapy frequently have lower body mass indices (BMIs), which in turn may increase their propensity for surgical complications following ITB pump placement. Although the most functional of patients with CP have close to normal growth, the subset of patients with $\mathrm{CP}$ where the level of impairment indicates ITB therapy consistently have growth below the 10th percentile of the general population. . $^{8,14}$ 
With these complications in mind, the surgical techniques for placing ITB pumps have been described in great detail. These techniques focus on placing the pumps below the fascia of the rectus abdominis muscle medially and the external oblique muscle laterally. ${ }^{2,13}$ Similarly, it has been argued that placing the spinal catheter and fascial connector in the lumbar subcutaneous fat can predispose catheter occlusion, migration, and other complications. ${ }^{3}$

Our group described a novel technique for paraspinal subfascial catheter placement. Of the 20 patients who underwent the procedure, none had hardware complications or CSF leakage that required replacement or revision over an average follow-up period of 5 months. ${ }^{5}$ This study expands on the original work by our group by following 43 patients who underwent paraspinal subfascial ITB placement with a median follow-up of 3.0 years.

\section{Methods \\ Study Review}

Using an institutional review board (IRB)-approved protocol, the electronic medical records were reviewed retrospectively for all patients who underwent paraspinal subfascial catheter placement by the senior author (D.H.H.) between July 2010 and February 2014. The IRB deemed that patient consent was not necessary for this study. Patients underwent paraspinal subfascial ITB placement in accordance with the protocol that was delineated previously by our group (2012). ${ }^{5}$ Patients referred for ITB pump placement are first seen by a physiatrist and a physical therapist for full assessment and to establish care. Spasticity is assessed and recorded. The patient is then scheduled for a trial ITB injection via lumbar puncture. The given dose is recorded, the patient is seen postinjection by a physical therapist again, and any improvement is documented and compared with pretrial baselines. If the patient is deemed to respond with good efficacy, he or she is then scheduled for pump implantation. Patients underwent follow-up by the surgeon at 2 weeks after the operation and were followed by their physiatrist thereafter. The physiatrists were part of the same hospital system as the neurosurgeon; as such, the neurosurgery team would be involved in following up with complications as they arose so long as the patient stayed with the physiatrist.

\section{Surgical Technique}

Patients are routinely positioned with their left side down in the lateral decubitus position. A 2-cm lumbar incision is planned over the L3-4 or L4-5 level on the posterior midline. An 8-cm incision is marked over the right abdomen approximately $3 \mathrm{~cm}$ below the costal margin.

The lumbar incision is opened down to the level of the muscular fascia. Approximately $1 \mathrm{~cm}$ to the right of midline, a $1.5-\mathrm{cm}$ incision is made in the fascia in a rostral to caudal direction. A 14-gauge Tuohy needle is introduced through this incision in order to access the thecal sac. If possible, only a single penetration is used since multiple dural punctures may increase the risk of CSF leakage. ${ }^{16}$ Once there is good CSF egress, the catheter is passed through the needle into the final desired position. Fluoroscopy may be avoided by measuring the catheter length preoperatively, but it can be useful to confirm the catheter position in cases of spinal deformity or other situations where accessing the subarachnoid space is more difficult.

The abdominal incision is opened, and the fascia of the rectus abdominis and external oblique muscles is also incised. At this point, a passer can be tunneled from the posterior incision in the subfascial space to the subfascial space anteriorly, which is a technique that was previously described by our group (2012). ${ }^{5}$ At this point, the pump catheter is passed to the lumbar incision, and the 2 catheters are trimmed and connected. The pump site catheter is pulled to remove any slack posteriorly until the connection is within the subfascial space that was dissected. A strain relief sleeve helps prevent catheter migration to the pump site. It should be verified that the CSF is flowing through the catheter at this point. Then, the catheter is connected to the pump, and the pump is placed into the subfascial pocket.

For closure, bacitracin irrigation is used, as well as betadine solution, at the skin edges. We also sprinkle dry vancomycin powder into the incisions and over the pump and catheter in order to prevent infection. The lumbar fascia can then be closed in a watertight fashion with a running suture. The fascia over the pump is also reapproximated in the abdominal incision. For both incisions, the deep dermal layers are closed with inverted interrupted Vicryl sutures followed by a running subcuticular Monocryl suture. The wounds are sealed with dermal glue, and sterile dressings applied.

\section{Results}

\section{Patient Characteristics}

Forty-three consecutive patients with ages ranging from 3 to 50 years (mean 16 years) underwent paraspinal subfascial ITB placement at our institution between July 2010 and February 2014. The mean BMI of our patient population was 18.6 (range 12.4-36.4). The mean time for surgery was 51 minutes from incision to the application of the dressing. The average length of stay after surgery was 2 days. The median follow-up of the patients in this study was 3.0 years (range $0.3-4.1$ years; mean 2.5 years), with only 3 of 43 patients having follow-ups shorter than 6 months and 30 patients having follow-ups longer than 2.0 years. Figure 1 summarizes the distribution of the patient follow-up lengths. Table 1 summarizes the demographic information for the patients in this study. Table 2 shows the indications for ITB placement.

\section{Complications}

Over the study duration, 12 of 43 patients had complications that warranted reoperation. Table 3 summarizes the indications for reoperation in these patients. Catheter malfunction $(n=4 ; 9.3 \%)$ and infection $(n=3 ; 7.0 \%)$ were the most common indications for reoperation. Of these 12 patients, $5(11.6 \%)$ patients had complications related to their lumbar incision or the lumbar portion of the catheter.

Among the patients with complications, the mean BMI was 17.2 (vs 19.2 for patients without complications). Of note, no patients had hardware erosions or CSF leaks during the study interval. It should be noted that 2 patients 


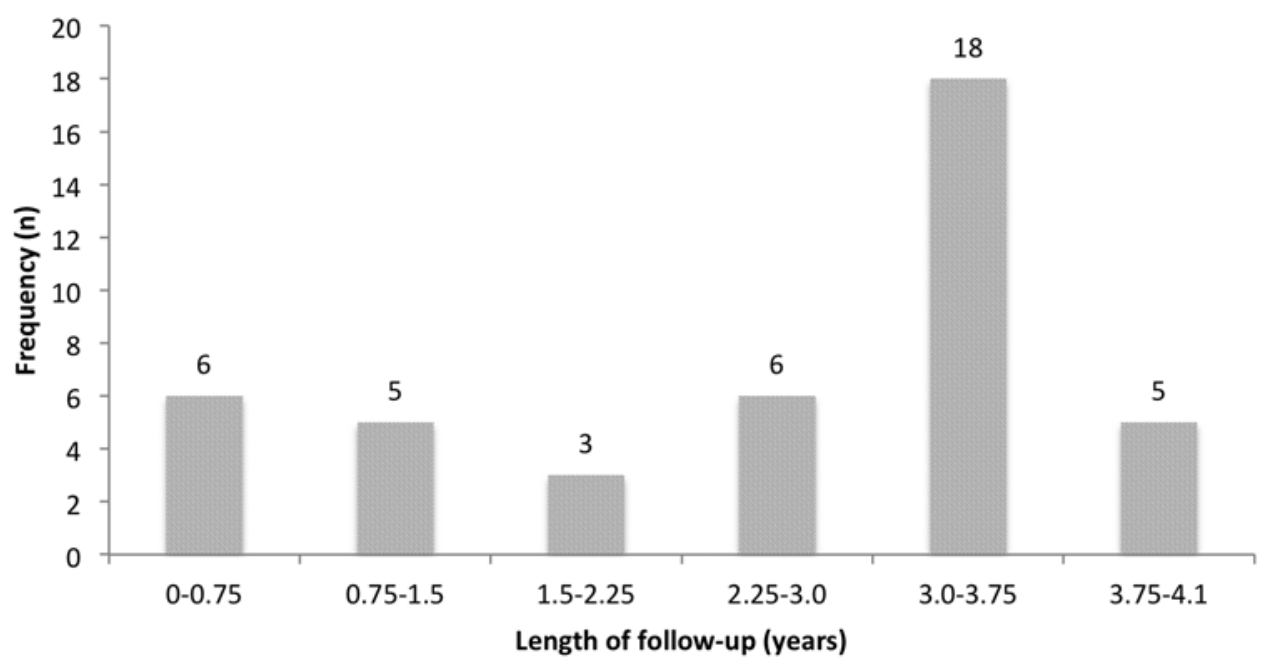

FIG. 1. Distribution of the follow-up lengths for the 43 patients. The median follow-up of the patients in this study was 3.0 years (range $0.3-4.1$ years; mean 2.5 years), with only 3 of 43 patients having follow-ups shorter than 6 months. Of note, 30 of 43 patients had follow-up periods over 2 years.

underwent revision with complete replacement of the system since no obvious site of malfunction could be isolated after intraoperative interrogation.

Of the 5 patients who developed complications at the lumbar incision or lumbar portion of the catheter, 2 complications were related to wound dehiscence and infection above the fascia. No CSF leakage was identified in either of these revisions, and no subfascial infection was identified. Another 2 patients developed catheter malfunctions and had abdominal radiographs that demonstrated looped catheters with kinks, thereby suggesting obstruction. These malfunctions were confirmed on revision and repaired. The remaining patient had a catheter disconnection at lumbar incision site, which was also repaired.

\section{Discussion}

This study is a follow-up to our prior study (2012), which demonstrated no hardware erosion, catheter migration, or CSF leakage in 20 patients who underwent a subfascial paraspinal ITB placement over a mean follow-up period of 5 months. As we noted previously, this shortterm complication rate was lower than that in a comparable series. ${ }^{5}$

This follow-up series demonstrated that patients with subfascial paraspinal placement continued to have no CSF leaks or hardware erosions over a longer follow-up period,

TABLE 1. Patient demographics

\begin{tabular}{cc}
\hline Variable & Value \\
\hline No. of patients & 43 \\
\hline Male & 24 \\
\hline Female & 19 \\
\hline Age at surgery in yrs & \\
\hline Mean & 16.52 \\
\hline Median & 13.86 \\
\hline Range & $3-50$ \\
\hline
\end{tabular}

and only 3 patients developed infections. Our group postulates that using a subfascial technique that permits closing the fascia over the catheter in a waterproof manner accounts for the decrease in CSF leakage. In contrast, a series by Motta et al. demonstrated that 20 of 200 consecutive patients $(10 \%)$ developed CSF leaks over a mean follow-up of 50 months, and 20 of 200 consecutive patients (10\%) developed infections. ${ }^{15}$ In a series by Ghosh et al. on patients treated by a single neurosurgeon over a period of 15 years, 9 of 119 patients developed CSF leaks or hardware erosions, and 26 of 119 patients developed infection..$^{10}$ In a series by Vender et al., ${ }^{17} 8.3 \%$ of patients who underwent ITB placement in either a subdermal pocket in adults, or in the fascia of the anterior rectus in children, developed CSF leaks over 5 years and less than 5\% developed infections. ${ }^{17}$ Comparatively, our series suggests that the subfascial paraspinal technique can reduce the risk of CSF leakage and catheter erosion in patients undergoing ITB placement.

In this study, the neurosurgeons followed their patients directly for only 2 weeks postoperatively. Following this 2-week window, the neurosurgeons depended on the physiatrist's notes to track complications. Of note, all patients included in this series were followed by physiatrists within the same medical system as the treating neurosurgery team, and none were referred to outside neurosurgeons for the management of complications.

TABLE 2. Indications for ITB placement

\begin{tabular}{lc}
\hline \multicolumn{1}{c}{ Variable } & No. of Patients \\
\hline Cerebral palsy & 33 \\
\hline Hereditary spastic paraplegia & 1 \\
\hline Multiple sclerosis & 3 \\
\hline Kernicterus & 1 \\
\hline Rett syndrome & 2 \\
\hline Spina bifida, tethered cord, \& diplegia & 1 \\
\hline Traumatic brain injury, spastic quadriplegia & 2 \\
\hline
\end{tabular}


TABLE 3. Indications for return to the operating room

\begin{tabular}{cc}
\hline Variable & No. of Complications \\
\hline Catheter malfunction & 4 \\
\hline Low flow & 1 \\
\hline Loop migration & 2 \\
\hline Catheter disconnection & 1 \\
\hline Infection & 3 \\
\hline Pump malfunction & 1 \\
\hline Lumbar wound dehiscence & 2 \\
\hline Poor response & 1 \\
\hline Unclear malfunction & 1 \\
\hline
\end{tabular}

\section{Conclusions}

The paraspinal subfascial technique for placing ITB catheters is a safe and effective method. In this long-term follow-up study, there were no instances of implant erosion or CSF leakage. The overall rate of complications requiring revision surgery was consistent with other similar series in the literature. While the previous short-term follow-up study demonstrated no catheter migration complications, this long-term follow-up study showed that this technique is not a fail safe for preventing these complications, and this can be an area for future investigation and innovation.

\section{References}

1. Albright AL, Barron WB, Fasick MP, Polinko P, Janosky $\mathrm{J}$ : Continuous intrathecal baclofen infusion for spasticity of cerebral origin. JAMA 270:2475-2477, 1993

2. Albright AL, Turner M, Pattisapu JV: Best-practice surgical techniques for intrathecal baclofen therapy. J Neurosurg 104 (4 Suppl):233-239, 2006

3. Ammar A, Ughratdar I, Sivakumar G, Vloeberghs MH: Intrathecal baclofen therapy-how we do it. J Neurosurg Pediatr 10:439-444, 2012

4. Armstrong RW, Steinbok P, Cochrane DD, Kube SD, Fife SE, Farrell K: Intrathecally administered baclofen for treatment of children with spasticity of cerebral origin. J Neurosurg 87:409-414, 1997

5. Bassani L, Harter DH: Paraspinal subfascial placement of lumbar intrathecal baclofen catheters: short-term outcomes of a novel technique. J Neurosurg Pediatr 9:93-98, 2012

6. Borowski A, Littleton AG, Borkhuu B, Presedo A, Shah S, Dabney KW, et al: Complications of intrathecal baclofen pump therapy in pediatric patients. J Pediatr Orthop 30:76-81, 2010

7. Butler C, Campbell S: Evidence of the effects of intrathecal baclofen for spastic and dystonic cerebral palsy. Dev Med Child Neurol 42:634-645, 2000

8. Day SM, Strauss DJ, Vachon PJ, Rosenbloom L, Shavelle RM, Wu YW: Growth patterns in a population of children and adolescents with cerebral palsy. Dev Med Child Neurol 49:167-171, 2007

9. Gerszten PC, Albright AL, Johnstone GF: Intrathecal baclofen infusion and subsequent orthopedic surgery in patients with spastic cerebral palsy. J Neurosurg 88:1009-1013, 1998

10. Ghosh D, Mainali G, Khera J, Luciano M: Complications of intrathecal baclofen pumps in children: experience from a tertiary care center. Pediatr Neurosurg 49:138-144, 2013

11. Gilmartin R, Bruce D, Storrs BB, Abbott R, Krach L, Ward $\mathrm{J}$, et al: Intrathecal baclofen for management of spastic cerebral palsy: multicenter trial. J Child Neurol 15:71-77, 2000

12. Gooch JL, Oberg WA, Grams B, Ward LA, Walker ML: Complications of intrathecal baclofen pumps in children. Pediatr Neurosurg 39:1-6, 2003

13. Kopell BH, Sala D, Doyle WK, Feldman DS, Wisoff JH, Weiner HL: Subfascial implantation of intrathecal baclofen pumps in children: technical note. Neurosurgery 49:753757, 2001

14. Krick J, Murphy-Miller P, Zeger S, Wright E: Pattern of growth in children with cerebral palsy. J Am Diet Assoc 96:680-685, 1996

15. Motta F, Buonaguro V, Stignani C: The use of intrathecal baclofen pump implants in children and adolescents: safety and complications in 200 consecutive cases. J Neurosurg 107 (1 Suppl):32-35, 2007

16. Seeberger MD, Kaufmann M, Staender S, Schneider M, Scheidegger D: Repeated dural punctures increase the incidence of postdural puncture headache. Anesth Analg 82:302-305, 1996

17. Vender JR, Hester S, Waller JL, Rekito A, Lee MR: Identification and management of intrathecal baclofen pump complications: a comparison of pediatric and adult patients. J Neurosurg 104 (1 Suppl):9-15, 2006

\section{Disclosures}

The authors report no conflict of interest concerning the materials or methods used in this study or the findings specified in this paper.

\section{Author Contributions}

Conception and design: Harter. Acquisition of data: Rubin. Analysis and interpretation of data: all authors. Drafting the article: Thakur. Critically revising the article: all authors. Reviewed submitted version of manuscript: all authors. Approved the final version of the manuscript on behalf of all authors: Harter. Administrative/technical/material support: Thakur, Rubin. Study supervision: Harter.

\section{Supplemental Information \\ Previous Presentations}

The materials in this manuscript were presented as a poster at the 2014 Pediatrics Section meeting of the AANS.

\section{Correspondence}

David H. Harter, 317 E. 34th St., Ste. 1002, New York, NY 10016. email: david.harter@nyumc.org. 